\title{
Effects of krill oil containing n-3 polyunsaturated fatty acids in phospholipid form on human brain function: a randomized controlled trial in healthy elderly volunteers
}

\author{
This article was published in the following Dove Press journal: \\ Clinical Interventions in Aging \\ 18 September 2013 \\ Number of times this article has been viewed
}

\author{
Chizuru Konagai ${ }^{1,2}$ \\ Kenichi Yanagimoto ${ }^{3}$ \\ Kohsuke Hayamizu ${ }^{3}$ \\ $\mathrm{Li} \mathrm{Han}{ }^{3}$ \\ Tomoko Tsuji ${ }^{3}$ \\ Yoshihiko Koga ${ }^{2}$ \\ 'Department of Food and Nutrition, \\ Japan Women's University, Bunkyo-ku, \\ Tokyo, Japan; ${ }^{2}$ Department of \\ Neuropsychiatry, Kyorin University \\ School of Medicine, Mitaka, Tokyo, \\ Japan; ${ }^{3}$ Human Life Science R\&D \\ Center, Nippon Suisan Kaisha Ltd, \\ Chiyoda-ku, Tokyo, Japan
}

\begin{abstract}
Background: Krill oil, rich in n-3 (omega-3) polyunsaturated fatty acids (PUFAs) incorporated in phosphatidylcholine, has been reported to have many effects on physiological function. However, there are few studies using psychophysiological methods published that describe the effects of krill oil on brain function. We investigated the influence of ingestion of krill oil on cognitive function in elderly subjects by using near-infrared spectroscopy and electroencephalography.
\end{abstract}

Methods: A randomized, double-blind, parallel-group comparative study design was adopted. Forty-five healthy elderly males aged 61-72 years were assigned to receive 12 weeks of treatment with: medium-chain triglycerides as placebo; krill oil, which is rich in n-3 PUFAs incorporated in phosphatidylcholine; or sardine oil, which is abundant in n-3 PUFAs incorporated in triglycerides. Changes in oxyhemoglobin concentrations in the cerebral cortex during memory and calculation tasks were measured. The P300 component of event-related potentials was also measured during a working memory task.

Results: During the working memory task, changes in oxyhemoglobin concentrations in the krill oil and sardine oil groups were significantly greater than those in the medium-chain triglyceride group at week 12. The differential value for P300 latency in the krill oil group was significantly lower than that in the medium-chain triglyceride group at week 12 . With regard to the calculation task, changes in oxyhemoglobin concentrations in the krill oil group were significantly greater than those in the medium-chain triglyceride group at week 12 .

Conclusion: This study provides evidence that n-3 PUFAs activate cognitive function in the elderly. This is especially the case with krill oil, in which the majority of n-3 PUFAs are incorporated into phosphatidylcholine, causing it to be more effective than sardine oil, in which n-3 PUFAs are present as triglycerides.

Keywords: eicosapentaenoic acid, docosahexaenoic acid, phosphatidylcholine, event-related potential, near-infrared spectroscopy, dorsolateral prefrontal cortex

\section{Introduction}

Researchers in a variety of areas have demonstrated that n-3 (omega-3) polyunsaturated fatty acids (PUFAs), including eicosapentaenoic acid (EPA; 20:5n-3) and docosahexaenoic acid (DHA; 22:6n-3), have considerable effects on physiological function. Animal and human studies ${ }^{1-4}$ in addition to epidemiological surveys ${ }^{5-7}$ have also shown that n-3 PUFAs effectively enhance and improve learning capability as well as memory and cognitive function. Moreover, intake of EPA and DHA is reported to reduce the risk of developing Alzheimer's disease. ${ }^{8}$ However, most studies in humans have assessed the effects of n-3 PUFAs based on the results of psychological tests and cognitive
Correspondence: Chizuru Konagai Department of Food and Nutrition, Japan Women's University, 2-8-I Mejirodai, Bunkyo-ku, Tokyo I I 2-868I, Japan

Tel +813598 I 3427

Fax +8I3 598I 3427

Email konagai@fc.jwu.ac.jp 
tasks. There are very few studies employing physiological methods that target elderly participants, although there are some studies targeting young adults or children. ${ }^{9-13}$

Fish oil contains abundant amounts of n-3 PUFAs, most of which is stored as triglycerides. Sardine oil is one of the most popular fish oils, and is abundant in n-3 PUFAs incorporated in triglycerides. In some cases, n-3 PUFAs are stored as phospholipids. Krill is the common name given to the order Euphausiacea of shrimp-like plankton. Krill oil is rich in n-3 PUFAs incorporated in phosphatidylcholine. Vakhapova et $\mathrm{al}^{14}$ and Richter et $\mathrm{al}^{15}$ demonstrated that phosphatidylserine containing n-3 PUFAs was effective for improvement of memory in the elderly. However, the differences in effects on cognitive function between these two forms of storage (ie, phospholipid and triglyceride), have not been clarified.

Among the neuroimaging methods available, near-infrared spectroscopy and event-related potentials are most suitable for measuring changes in brain function longitudinally and noninvasively. Moreover, the devices required are easy to operate and the cost is low compared with other neurophysiological methods. When brain activity is activated locally, the supply of oxygen to that area increases accordingly. Oxygen binds with hemoglobin and is transported in the form of oxyhemoglobin. Therefore, by measuring blood oxyhemoglobin concentrations using near-infrared spectroscopy, it is possible to identify the level of activation of regional cerebral function resulting from the execution of tasks. Near-infrared spectroscopy emits near-infrared light from above the scalp. Analyzing near-infrared light at several wavelengths detected after it has been absorbed by oxyhemoglobin while passing through the tissues of the cerebral cortex makes it possible to measure the relative changes in oxyhemoglobin concentration as a marker of neural activity in the cerebral cortex. ${ }^{16}$ Event-related potentials, obtained by analyzing electroencephalograms (EEGs), represent information processing in the brain electrophysiologically, and are elicited especially while executing cognitive tasks. Among the event-related potential components, $\mathrm{P} 300$ is considered to reflect cognitive processes, such as making decisions and controlling behaviors and activities. ${ }^{17}$ Because near-infrared spectroscopy has a relatively high spatial resolution, it is useful for localizing activated areas. The event-related potential, in contrast, has the advantage of high temporal resolution.

In this study, two types of continuous performance tasks were used during measurement of changes in oxyhemoglobin concentration and event-related potentials, ie, the working memory task and the calculation task. The amount of working memory mobilized to perform each task is considerably different. The former needs mobilization of much working memory. On the contrary, the latter demands little working memory. Working memory is a function that serves as the basis of cognitive activity.

Prior to conducting a study with a large sample size, we performed a preliminary examination to clarify the effects of long-term ingestion of krill oil and sardine oil on cognitive function in elderly males using the two neurophysiological methods described previously. Further, we investigated differences in effects of n-3 PUFAs on cognitive function in relation to the different chemical forms of storage. Only male subjects were recruited to participate in this study, because cerebral blood volume changes during cognitive activation have been found to be gender-dependent. ${ }^{18}$ Differences in event-related potentials have also been reported to be gender-dependent. ${ }^{19}$

\section{Materials and methods Subjects}

Healthy male subjects in their 60 s and 70 s who had retired from employment in the Japanese business sector were recruited for this study; all participants were paid volunteers. Eligibility for participation was as follows: right-handedness, not requiring prescription medication, no history of mental disorders or cerebrovascular disease, absence of serious liver, kidney, heart, respiratory, endocrine, or metabolic diseases, and absence of food allergies to fish or crustaceans. The participants were clinically confirmed by a psychiatrist to have no cognitive impairment.

We provided written and oral explanations in advance about the purpose of our study in accordance with the Declaration of Helsinki. All the participants gave their written informed consent to participate in the study protocol, which was approved by the ethics committee of Kyorin University School of Medicine.

The participants were measured for their height, body weight, and blood pressure, and then underwent a blood test (white blood cell count, red blood cell count, hemoglobin, and hematocrit), a serum chemistry test (aspartate aminotransferase, alanine aminotransferase, gamma-glutamyl transpeptidase, creatinine, total cholesterol, high-density lipoprotein cholesterol, low-density lipoprotein cholesterol, triglycerides, blood glucose, glycosylated hemoglobin, and uric acid) and urinalysis (occult blood, urinary protein, urinary glucose, and urobilinogen). Forty-five men of mean age ( \pm standard deviation) $67.1 \pm 3.4$ years with no markedly abnormal findings for these parameters were randomly divided into three treatment groups ( $\mathrm{n}=15$ per group). The participants were 
instructed to avoid excessive consumption of alcohol and food, to limit strenuous exercise, and to refrain from taking supplements that contained PUFAs during the study.

\section{Experimental supplement}

Three types of experimental supplement were used: krill oil as a typical oil rich in PUFAs incorporated in phosphatidylcholine, sardine oil as a typical fish oil, and medium-chain triglycerides as placebo. Each supplement was prepared by Nippon Suisan Kaisha Ltd. Table 1 shows the composition of the main ingredients in the supplements. They were supplied in soft gelatin capsules containing $0.25 \mathrm{~g}$ oil per capsule.

Table I Composition of each supplement (per daily dose)

\begin{tabular}{|c|c|c|c|}
\hline Nutrients & MCTs & KO & so \\
\hline \multicolumn{4}{|l|}{ Components } \\
\hline Water (g) & $<0.02$ & Trace & 0 \\
\hline Total lipids (g) & 1.98 & 1.98 & 1.98 \\
\hline Tocopherol (mg) & 0 & 14 & 20 \\
\hline \multicolumn{4}{|l|}{ Lipid class } \\
\hline Phospholipids in total lipids (g) & 0 & 0.90 & 0 \\
\hline \multicolumn{4}{|l|}{ Fatty acid composition of total lipids } \\
\hline $8: 0(\mathrm{mg})$ & $\mathrm{I}, 497$ & 13 & 0 \\
\hline $10: 0$ (mg) & 287 & 7 & 0 \\
\hline 12:0 (mg) & 5 & 5 & 0 \\
\hline I $4: 0$ (mg) & 0 & 155 & 84 \\
\hline I4:I (mg) & 0 & 4 & 0 \\
\hline 15:0 (mg) & 0 & 7 & 3 \\
\hline 16:0 (mg) & 0 & 280 & 106 \\
\hline $16: 1$ (mg) & 0 & 87 & 148 \\
\hline $16: 2(\mathrm{mg})$ & 0 & 9 & 27 \\
\hline $16: 3(\mathrm{mg})$ & 0 & 3 & 44 \\
\hline $16: 4(\mathrm{mg})$ & 0 & 11 & 77 \\
\hline 17:0 (mg) & 0 & 35 & 5 \\
\hline 17:I (mg) & 0 & 4 & 0 \\
\hline I8:0 (mg) & 0 & 19 & 9 \\
\hline |8:। (mg) & 13 & 249 & 154 \\
\hline $18: 2 n-6(m g)$ & 4 & 25 & 22 \\
\hline $18: 3 n-3(\mathrm{mg})$ & 0 & 13 & 14 \\
\hline $18: 4 n-3(m g)$ & 0 & 34 & 82 \\
\hline 20:1 (mg) & 0 & 11 & 3 \\
\hline $20: 2 n-6(m g)$ & 0 & 0 & 2 \\
\hline $20: 3 n-6(m g)$ & 0 & 0 & 3 \\
\hline $20: 4 n-6(m g)$ & 0 & 3 & 20 \\
\hline $20: 4 n-3(m g)$ & 0 & 4 & 17 \\
\hline $20: 5 n-3(E P A)(m g)$ & 0 & 193 & 491 \\
\hline $21: 5 n-3(m g)$ & 0 & 5 & 19 \\
\hline $22: 0(\mathrm{mg})$ & 0 & 0 & 3 \\
\hline $22: 1$ (mg) & 0 & 12 & 0 \\
\hline $22: 5 n-6(m g)$ & 0 & 0 & 5 \\
\hline $22: 5 n-3(m g)$ & 0 & 5 & 46 \\
\hline 22:6n-3 (DHA) (mg) & 0 & 92 & 251 \\
\hline Unidentifiable (mg) & 0 & 70 & 54 \\
\hline
\end{tabular}

Note: Subjects took four capsules $(0.25 \mathrm{~g}$ oil/capsule) twice a day after breakfast and dinner.

Abbreviations: MCTs, medium-chain triglycerides; $\mathrm{KO}$, krill oil; SO, sardine oil; EPA, eicosapentaenoic acid; DHA, docosahexaenoic acid.
There was no difference in color, size, form, smell, or taste between the three types of capsules. Subjects were asked to take four capsules twice a day after breakfast and dinner for 12 weeks (eight capsules daily), from the day following the initial measurements to the day of the final measurements. The participants were instructed to complete a self-recorded checklist to document their supplement intake during the study period.

\section{Experimental design}

A randomized, double-blind, parallel-group, comparative study design was adopted. A computer-generated random allocation sequence was used for participant randomization. This was performed by a person who was not involved with other aspects of the study. Information about assignment was not revealed to the researchers until the key was opened. The participants were not informed about which treatment was included in their capsules. Before the participants started treatment, and at week 6 and week 12, we simultaneously recorded changes in oxyhemoglobin concentration and an EEG during performance of the working memory task, and measured changes in oxyhemoglobin concentration during the calculation task. Near-infrared spectroscopy and EEG recordings were performed in an electrically shielded chamber in the laboratory of the neuropsychiatry division at Kyorin University. The following were also performed: a survey of food intake frequency, measurements of body weight and blood pressure, a blood test, a serum chemistry test, measurements of plasma fatty acids (dihomo-gammalinolenic acid, arachidonic acid, EPA, and DHA), and urinalysis. Subjects showing any abnormal physical symptoms or findings on blood or urine testing during treatment were withdrawn from the study.

\section{Food frequency method}

The Food Frequency Questionnaire Based on Food Groups (FFQg, version 3.0, Kenpakusha, Tokyo, Japan) was used to evaluate intake of nutrients during the study period. The FFQg was developed for use in Japan and is based on 29 food groups and 10 types of cooking methods, and is used for estimating energy and nutrient intake per week for the previous month. ${ }^{20}$ From the FFQg, the mean dietary energy intake was calculated according to the fifth revised and enlarged edition of the Standard Tables of Food Composition in Japan. ${ }^{21}$ The participants' responses were obtained by interview. We calculated the intake of energy and of individual nutrients per day (protein, fats, carbohydrates, sodium, potassium, calcium, magnesium, 
phosphorus, iron, zinc, copper, manganese, dietary fiber, retinol equivalent, vitamin $\mathrm{D}$, alpha-tocopherol, vitamin $\mathrm{K}$, vitamin $\mathrm{B}_{1}$, vitamin $\mathrm{B}_{2}$, niacin, vitamin $\mathrm{B}_{6}$, vitamin $\mathrm{B}_{12}$, folic acid, pantothenic acid, vitamin $C$, saturated fatty acids, monounsaturated fatty acids, PUFAs, n-3 PUFAs, n-6 PUFAs, and cholesterol).

\section{Tasks}

\section{Working memory task}

A continuous performance task using the 2-back paradigm was carried out. Numerals from 1 to 9 randomly appeared successively on a computer screen placed $150 \mathrm{~cm}$ in front of the participant for $500 \mathrm{msec}$ each, with an interstimulus interval of 2,250 msec. The participants were instructed to push a button with their right thumb when the numeral " 3 " was displayed only if they recalled that the numeral 2-back was even, and to do nothing when the numeral 2-back was odd. Two hundred numerals were presented in total, with the target " 3 " numeral being shown at a rate of $10 \%$ (20 trials). For 14 seconds before starting to show the stimulus, the numeral " 0 " was shown on the display monitor for the same duration and interval as the stimulus, and the participants were instructed to watch the numeral carefully. The task lasted 450 seconds.

\section{Calculation task}

A calculation task was conducted using the Uchida-Kraepelin test paper. The participants were asked to add two adjacent numerals and write down only the last numeral of the two-digit number that was obtained. The participants were instructed to make the calculation as quickly and accurately as possible. The task lasted 300 seconds.

\section{Measurement and data analysis \\ Changes in oxyhemoglobin concentration}

Changes in oxyhemoglobin concentration while participants performed the tasks were measured using near-infrared spectroscopy (Optical Topography System ETG-4000, Hitachi Medical Co, Tokyo, Japan). We used a two-sided $3 \times 3$ probe holder, and placed the emitter probes and detector probes at sites where we could measure changes in oxyhemoglobin concentration in each participant's bilateral dorsolateral prefrontal cortex. That is, the front row inner optodes were positioned symmetrically $0.5 \mathrm{~cm}$ outside of $\mathrm{Fp}_{1}$ or $\mathrm{Fp}_{2}$ according to the International EEG 10-20 system, and changes in each participant's oxyhemoglobin concentration were measured from a total of 24 channels (Figure 1). Mean changes in oxyhemoglobin concentration were calculated against

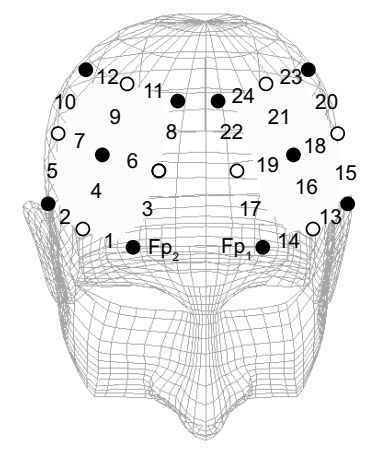

Figure I Channel positions for near-infrared spectroscopy. Changes in oxyhemoglobin concentration were measured at 24 sites in the bilateral frontal areas of the brain. Filled (black) circles and open (white) circles show the emitter and detector probes, respectively. The numbers show the location of the measured channels.

the mean concentration during a 10 -second period for the prestimulus baseline.

\section{Electroencephalography}

While the participants performed the working memory task, we simultaneously measured changes in oxyhemoglobin concentration and event-related potentials. $\mathrm{Ag} / \mathrm{AgCl}$ electrodes were located at $\mathrm{Cz}$ (vertex) and $\mathrm{Pz}$ (midline parietal site) according to the International EEG 10-20 system. The EEG was recorded and filtered from $0.1 \mathrm{~Hz}$ to $100 \mathrm{~Hz}$, using linked electrodes at the earlobes as the reference. The EEG was recorded for 450 seconds while the participants performed the working memory task. The EEG was averaged off-line for a period of $1,000 \mathrm{msec}$, beginning $100 \mathrm{msec}$ prior to the stimulus onset. The baseline was corrected to the mean value of the voltage during a period of $100 \mathrm{msec}$ prestimulus. From ten to 20 trials without artifacts due to eye blinking or body movement were averaged to obtain the event-related potential waveform. The peak latency and amplitude of the P300 component of the event-related potential waveform at $\mathrm{Cz}$ and $\mathrm{Pz}$ were measured. The differential values of P300 latency and amplitude at week 6 and week 12 compared with those at week 0 were calculated for both sites. P300 is the positive potential that appears approximately $300 \mathrm{msec}$ after a stimulus is presented.

\section{Statistical analysis}

Statistical Package for the Social Sciences version 16.0 software (SPSS Inc., Chicago, IL, USA) was used for statistical processing. Two-way repeated-measures analysis of variance with the Bonferroni post hoc test was carried out on the following near-infrared spectroscopic and other data: change in oxyhemoglobin concentration, body weight, body mass index, blood pressure, blood test values, serum 
chemistry test values, urinalysis, nutritional intake based on food frequency method, and plasma fatty acid concentration. For P300 latency and amplitude, the Kruskal-Wallis test was used to analyze the differential values measured at week 6 and week 12 compared with those at week 0 . The MannWhitney $U$-test with Bonferroni correction was then used to determine any differences between the treatments. The statistical significance level was set at $P<0.05$.

\section{Results}

\section{Participants}

A flow chart for study participation is shown in Figure 2. As mentioned earlier, 45 males who met the inclusion criteria agreed to participate in this study. They were randomly assigned to three treatment groups. Age, height, body weight, body mass index, smoking status, and alcohol consumption were compared by one-way analysis of variance or the chisquare test. No significant differences were found in any of the items except for height $(F[2,42]=4.379, P=0.019$ by one-way analysis of variance) between the three groups (Table 2).

Three of the 45 participants dropped out during the study period. Data for 15 participants in the medium-chain triglyceride group, 13 in the krill oil group, and 14 in the sardine oil group were used for analysis. The reasons for dropping out were epigastric pain (two participants) and dermatitis (one participant). These cases were judged not to be directly related to ingestion of supplements.

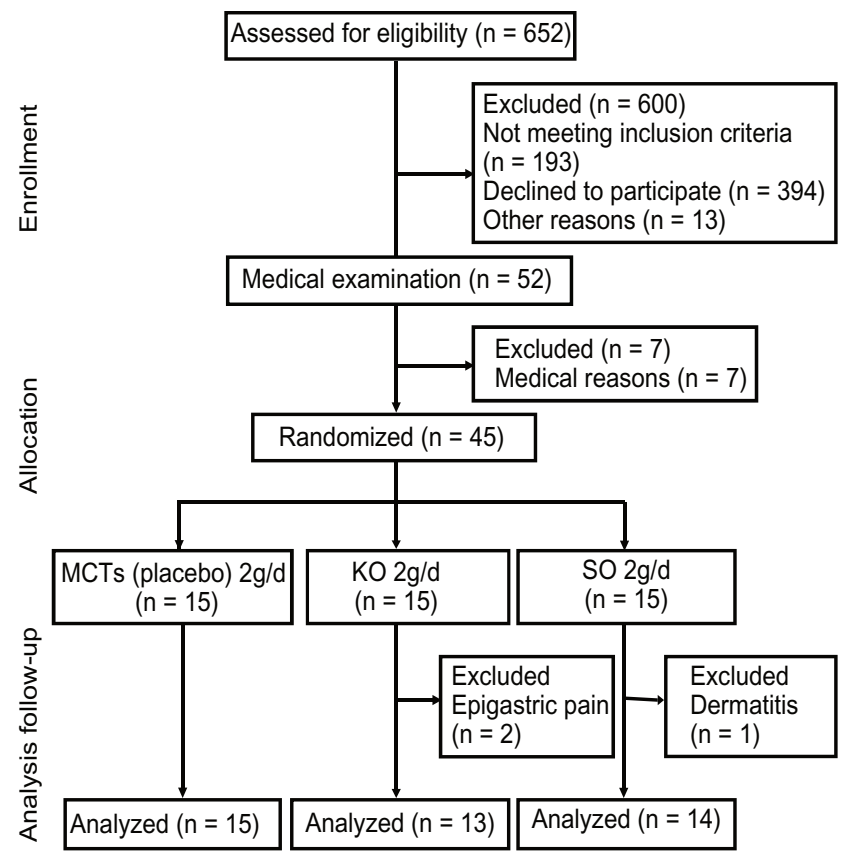

Figure 2 Flow chart of participation throughout the study. Abbreviations: MCTs, medium-chain triglycerides; KO, krill oil; SO, sardine oil.
Table 2 Baseline characteristics of the participants

\begin{tabular}{lllll}
\hline & $\begin{array}{l}\text { MCT } \\
(\mathbf{n}=15)\end{array}$ & $\begin{array}{l}\text { KO } \\
(\mathbf{n}=15)\end{array}$ & $\begin{array}{l}\text { SO } \\
(\mathbf{n}=15)\end{array}$ & P-value \\
\hline Age (years) & $67.3 \pm 3.4$ & $67.1 \pm 3.7$ & $67.0 \pm 3.3$ & 0.962 \\
Height $(\mathrm{cm})$ & $167.0 \pm 3.9$ & $163.5 \pm 5.8$ & $168.6 \pm 4.5$ & 0.019 \\
Body weight $(\mathrm{kg})$ & $66.2 \pm 10.7$ & $64.9 \pm 8.3$ & $63.0 \pm 5.9$ & 0.604 \\
BMI $\left(\mathrm{kg} / \mathrm{m}^{2}\right)$ & $23.7 \pm 3.5$ & $22.8 \pm 2.6$ & $23.6 \pm 2.2$ & 0.666 \\
Current smoker & $\mathrm{I}(7)$ & $2(13)$ & $4(27)$ & 0.306 \\
Alcohol consumer & $\mathrm{II}(73)$ & $13(87)$ & $12(80)$ & 0.659 \\
\hline
\end{tabular}

Notes: Mean \pm standard deviation or $n(\%)$. P-values derived by one-way analysis of variance or chi-square test.

Abbreviations: MCT, medium-chain triglyceride; KO, krill oil; SO, sardine oil; BMI, body mass index.

\section{Supplement intake rate}

According to the self-recording checklist, mean supplement intake for the experimental period (excluding dropout participants) was $98.2 \% \pm 2.4 \%$ in the medium-chain triglyceride group $(\mathrm{n}=15), 98.7 \% \pm 1.6 \%$ in the krill oil group $(n=13)$, and $98.5 \% \pm 1.7 \%$ in the sardine oil group $(n=14)$. No significant difference in intake was found between the three groups $(F[2,39]=0.181, P=0.835$ by one-way analysis of variance).

\section{Food frequency questionnaire, vital signs, and blood and urine tests}

No significant differences between the treatments or ingestion periods were found with regard to any of the nutrients measured by the food frequency method, such as amounts of major components ingested or dietary fatty acids and cholesterol. No harmful events due to the treatments were observed in any of the treatment groups during the study. None of the groups showed any significant variations in body weight, body mass index, or blood pressure during the study period. Moreover, no changes attributable to ingestion of the supplements were observed in blood tests, serum chemistry tests, or urinalysis.

\section{Plasma fatty acids}

With regard to the concentration of PUFAs in plasma (Table 3), significant interactions were found between the treatments and ingestion periods in terms of dihomogamma-linolenic acid concentration $(F[4,78]=3.516$, $P=0.011$ by two-way repeated-measures analysis of variance) and EPA concentration $(F[4,78]=5.141, P=0.001$ by two-way repeated-measures analysis of variance). Further analysis using Bonferroni post hoc testing showed that the EPA concentration in the sardine oil group was higher than that in the medium-chain triglyceride group at week $12(P=0.045)$. 
Table 3 Polyunsaturated fatty acids in plasma

\begin{tabular}{|c|c|c|c|c|c|c|c|c|c|c|}
\hline & \multicolumn{3}{|l|}{ Week 0} & \multicolumn{3}{|l|}{ Week 6} & \multicolumn{3}{|l|}{ Week I 2} & \multirow[t]{2}{*}{$P$-value } \\
\hline & MCT & KO & so & MCT & KO & so & MCT & KO & so & \\
\hline $\begin{array}{l}\text { DGLA } \\
(\mu g / \mathrm{mL})\end{array}$ & $25.3 \pm 2.5$ & $21.1 \pm 2.6$ & $27.3 \pm 2.1$ & $25.4 \pm 2.1$ & $19.5 \pm 1.8$ & $23.4 \pm 1.8$ & $25.8 \pm 2.4$ & $19.9 \pm 2.1$ & $20.0 \pm 2.0$ & 0.011 \\
\hline $\begin{array}{l}\text { AA } \\
(\mu g / m L)\end{array}$ & $156 \pm 8$ & $130 \pm 5$ & $143 \pm 8$ & $158 \pm 8$ & $136 \pm 6$ & $152 \pm 7$ & $159 \pm 10$ & $128 \pm 7$ & $|4| \pm 7$ & 0.700 \\
\hline $\begin{array}{l}\text { EPA } \\
(\mu g / \mathrm{mL})\end{array}$ & $110 \pm 10$ & $122 \pm 20$ & $99 \pm 10$ & $113 \pm 12$ & $155 \pm 2 \mid$ & $155 \pm 14$ & $108 \pm 12^{a}$ & $125 \pm 18^{\mathrm{a}, \mathrm{b}}$ & $157 \pm 12^{b}$ & 0.001 \\
\hline $\begin{array}{l}\text { DHA } \\
(\mu g / m L)\end{array}$ & $192 \pm 14$ & $191 \pm 19$ & $191 \pm 16$ & $196 \pm 14$ & $196 \pm 17$ & $205 \pm 15$ & $177 \pm 13$ & $179 \pm 19$ & $187 \pm 15$ & 0.944 \\
\hline
\end{tabular}

Notes: Mean \pm standard error. MCT, $n=15 ; \mathrm{KO}, \mathrm{n}=13 ; \mathrm{SO}, \mathrm{n}=14$. $P$-values show significant probability of interaction between treatments and ingestion periods derived by two-way repeated-measures analysis of variance. Numerical values within the same period given different superscript letters ( ${ }^{\mathrm{a}}$ and $\left.{ }^{\mathrm{b}}\right)$ show significant differences between treatments $(P<0.05$ by Bonferroni post hoc test).

Abbreviations: DGLA, dihomo-gamma-linolenic acid (20:3n-6); AA, arachidonic acid (20:4n-6); EPA, eicosapentaenoic acid (20:5n-3); DHA, docosahexaenoic acid (22:6n-3); MCT, medium-chain triglyceride; $\mathrm{KO}$, krill oil; $\mathrm{SO}$, sardine oil.

\section{Working memory task}

\section{Changes in oxyhemoglobin concentration}

Figure 3 shows a comparison of changes in oxyhemoglobin concentration at 225.0 seconds after the start of executing the task. With regard to changes in oxyhemoglobin concentration in response to performance of the working memory task, the results of two-way repeated-measures analysis of variance showed a significant interaction in channel $10(F[4,78]=4.331$, $P=0.003$ by two-way repeated-measures analysis of variance).
Compared with the medium-chain triglyceride group, the sardine oil group $(P=0.043)$ and the krill oil group $(P=0.004)$ showed significantly greater changes in oxyhemoglobin concentrations at week 12 (Figure 4).

\section{Event-related potentials}

Figure 5 shows the changes in P300 latency and amplitude at $\mathrm{Cz}$ and $\mathrm{Pz}$. There were slight differences in the differential values for P300 latency between the treatments at week 6

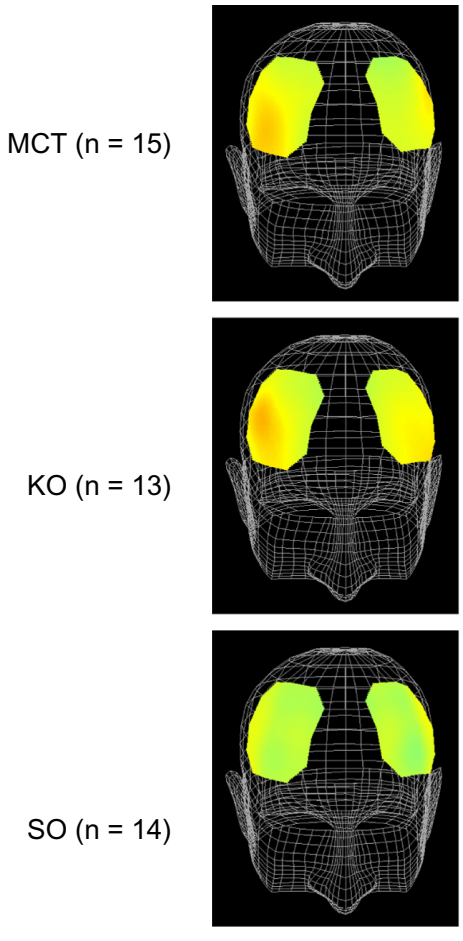

Week 0
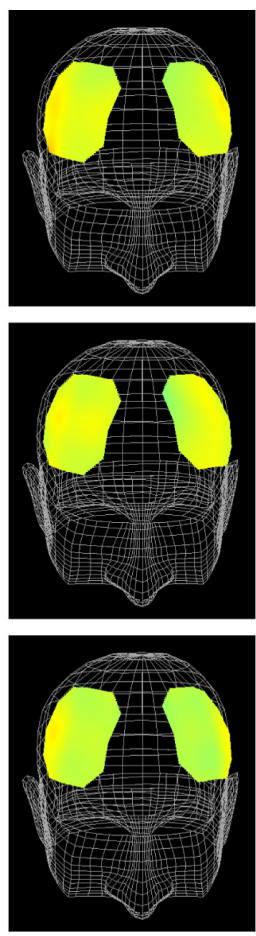

Week 6
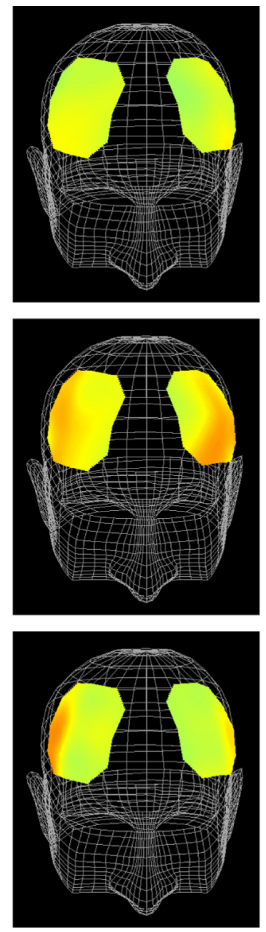

Week 12
$[\mathrm{mM} \times \mathrm{mm}]$

0.55

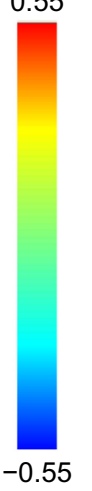

Figure 3 Topographic maps of changes in oxyhemoglobin concentration at 225.0 seconds during working memory task.

Note: MCT, $n=15 ; \mathrm{KO}, \mathrm{n}=13$; SO, $\mathrm{n}=14$.

Abbreviations: $\mathrm{MCT}$, medium-chain triglyceride; KO, krill oil; SO, sardine oil. 


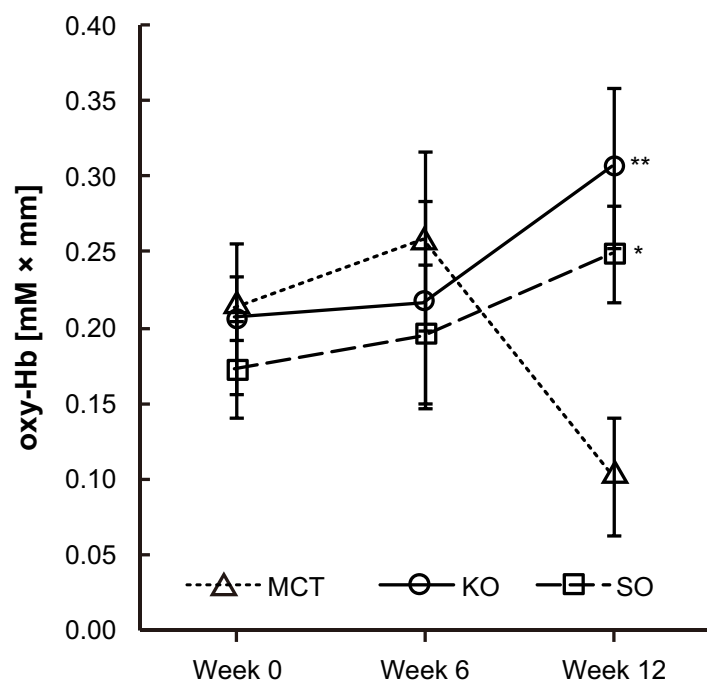

Figure 4 Comparison of changes in oxyhemoglobin (oxy- $\mathrm{Hb}$ ) concentrations at channel 10 during working memory task.

Notes: Values are expressed as the group mean \pm standard error. MCT, $n=15$; $K O, n=13$; SO, $n=14$. Values were significantly different from the MCT group. $* P<0.05$, ${ }^{*} * P<0.01$ by Bonferroni post hoc test.

Abbreviations: MCT, medium-chain triglyceride; KO, krill oil; SO, sardine oil.
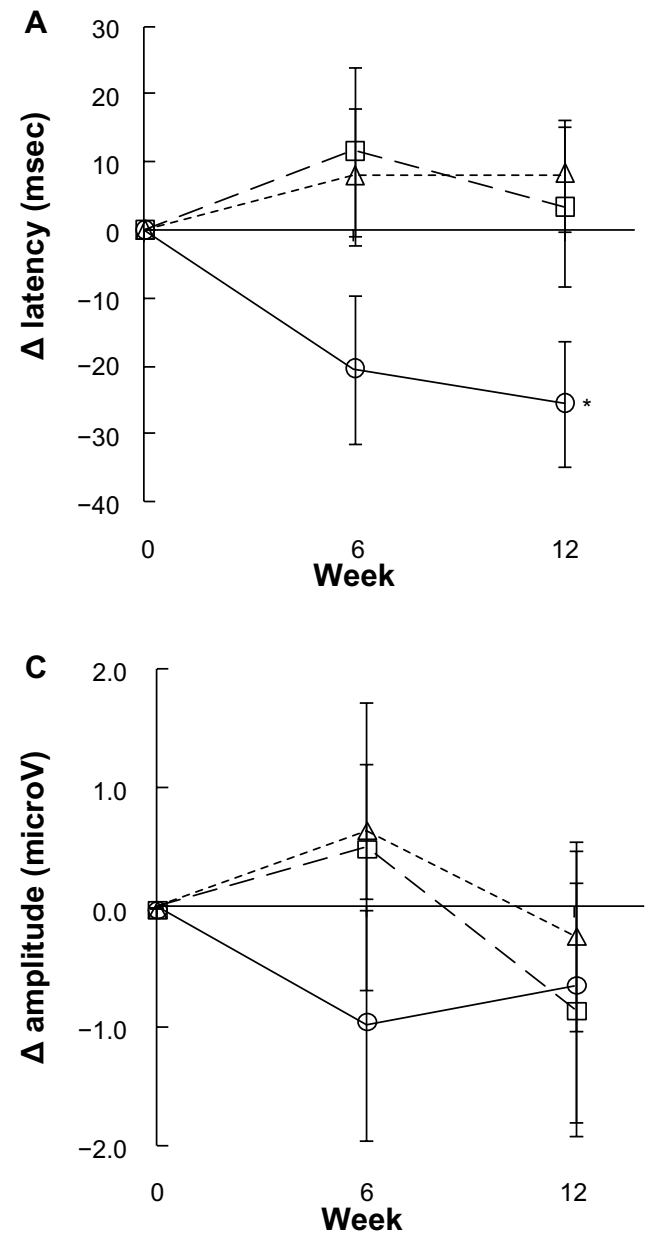

$\left(\mathrm{Cz}, \chi^{2}=4.981, d f=2, P=0.083 ; \mathrm{Pz}, \chi^{2}=5.130, d f=2\right.$, $P=0.077$, Kruskal-Wallis test). At week 12 of the ingestion period, there were differences between the groups $(\mathrm{Cz}$, $\chi^{2}=6.118, d f=2, P=0.047 ; \mathrm{Pz}, \chi^{2}=5.754, d f=2, P=0.056$, Kruskal-Wallis test), and a significant difference was observed between the krill oil and medium-chain triglyceride groups $(\mathrm{Cz}, P=0.027$ and $\mathrm{Pz}, P=0.030$, Mann-Whitney $U$-test with Bonferroni correction). Both sites showed no significant difference between treatments for differential values in P300 amplitude at either week 6 or week 12.

\section{Calculation task}

Figure 6 shows the mean changes in oxyhemoglobin concentration at 150.0 seconds after starting the task. For changes in oxyhemoglobin concentration in response to execution of the calculation task, the results of two-way repeated-measures analysis of variance showed a slight interaction between the treatments and ingestion periods at
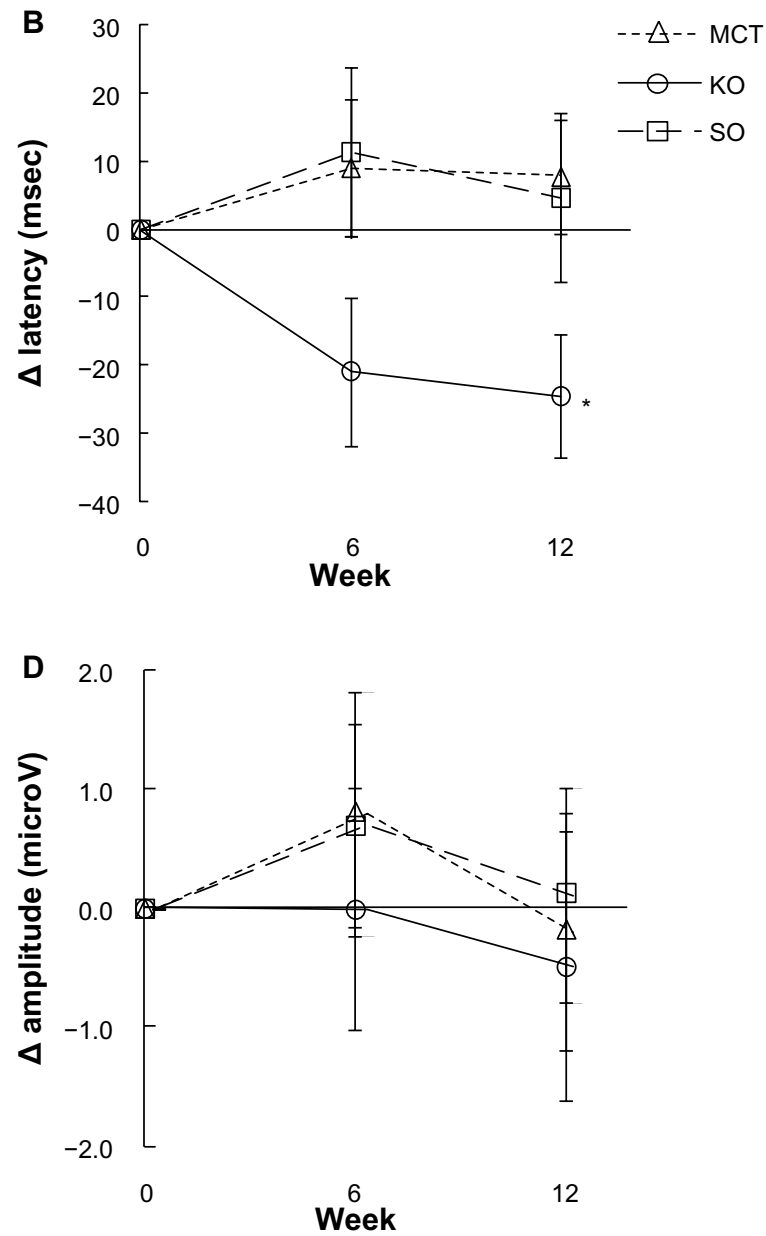

Figure 5 Comparison of differential values in P300 latencies and amplitudes during the working memory task. (A) Latency at Cz, (B) latency at Pz, (C) amplitude at Cz, and (D) amplitude at Pz.

Notes: Values are expressed as the group mean \pm standard error. MCT, $\mathrm{n}=15 ; \mathrm{KO}, \mathrm{n}=13 ; \mathrm{SO}, \mathrm{n}=14$. Values were significantly different from the MCT group: $* \mathrm{P}<0.05$ by Mann-Whitney U-test with Bonferroni correction. No significant difference in P300 amplitude was observed between the treatments at week 6 or week 12 . Abbreviations: MCT, medium-chain triglyceride; KO, krill oil; SO, sardine oil. 


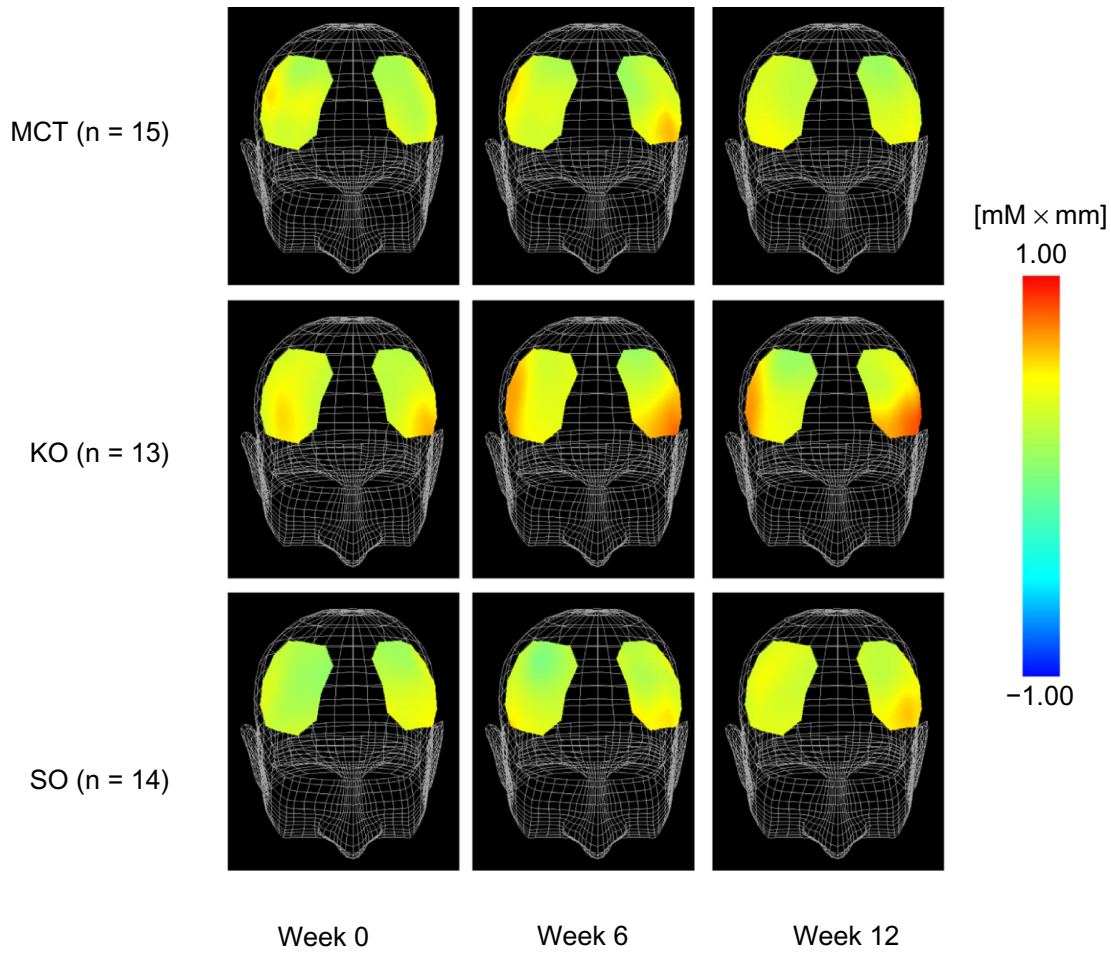

Figure 6 Topographic maps of changes in oxyhemoglobin concentration at 150.0 seconds during the calculation task.

Note: $\mathrm{MCT}, \mathrm{n}=15 ; \mathrm{KO}, \mathrm{n}=13$; $\mathrm{SO}, \mathrm{n}=14$.

Abbreviations: MCT, medium-chain triglyceride; KO, krill oil; SO, sardine oil.

channel $15(F[4,78]=2.236, P=0.073$ by two-way repeatedmeasures analysis of variance). The krill oil group showed significantly greater changes in oxyhemoglobin concentration compared with the medium-chain triglyceride group at week $12(P=0.006$, Figure 7$)$.

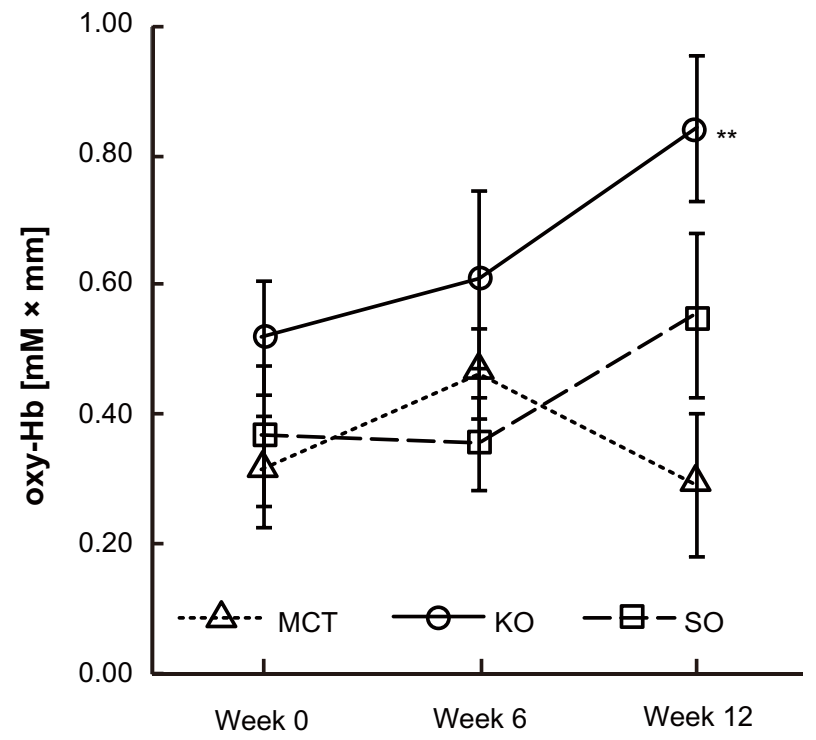

Figure 7 Comparison of changes in oxyhemoglobin (oxy-Hb) concentration at channel 15 during the calculation task.

Notes: Values are expressed as the group mean \pm standard error. MCT, $\mathrm{n}=15$; $K O, n=13$; SO, $n=14$. Values were significantly different from the MCT group: $* * P<0.0$ l by Bonferroni post hoc tests.

Abbreviations: MCT, medium-chain triglyceride; KO, krill oil; SO, sardine oil.

\section{Discussion}

The results of the working memory tasks show that the groups taking krill oil or sardine oil for 12 weeks had greater changes in oxyhemoglobin concentration in channel 10 than the group taking medium-chain triglycerides. With regard to the effect of n-3 PUFAs, Jackson et al have already demonstrated in their pilot study using near-infrared spectroscopy that supplementation with DHA-rich fish oil, in comparison with placebo, resulted in significantly increased oxyhemoglobin concentrations in the prefrontal areas in healthy young adults. ${ }^{13}$ As the function of the cerebral cortex declines with age, a smaller increase is observed in oxyhemoglobin concentrations resulting from execution of cognitive tasks. ${ }^{18,22}$ Near-infrared spectroscopy probes including channel 10 were placed to measure the function of the dorsolateral prefrontal cortex in the present study. The dorsolateral prefrontal cortex performs a variety of functions. Among these, mobilization of working memory is one of the important functions of the dorsolateral prefrontal cortex. ${ }^{23-25}$ Aging reduces regional cerebral blood flow, which causes deterioration of cerebral function. ${ }^{26}$ Aging has also been shown to reduce the level of dorsolateral prefrontal cortex activity during execution of working memory tasks. ${ }^{27}$ The results of this study suggest that long-term ingestion of krill oil and sardine oil promotes working memory function by activating the dorsolateral prefrontal 
cortex in elderly people, and thus prevents deterioration in cognitive activity.

Very few studies have been published using the eventrelated potential to identify the effects of EPA and DHA. Fontani et al compared event-related potential measurements while subjects executed the Go/No-Go paradigm before and after taking fish oil for 35 days, and reported improvement in attentional functions after ingestion of n-3 PUFAs. ${ }^{9}$ Regarding P300, which was measured simultaneously with near-infrared spectroscopy recording, we observed no differences in amplitude between our treatment groups, although the group that took krill oil for 12 weeks showed a significant decrease in latency compared with the medium-chain triglyceride group. P300 latency is thought to reflect the rate of information processing, while amplitude is thought to reflect the amount of mobilization of processing resources. ${ }^{28}$ Continuous ingestion of krill oil was thus shown to expedite the information processing rate. $\mathrm{P} 300$ latency is known to be prolonged with aging, ${ }^{29,30}$ ie, ingestion of krill oil is likely to ameliorate the reduction of cerebral function associated with aging, or to maintain such function.

During the calculation tasks, the krill oil group showed significantly greater changes in oxyhemoglobin concentrations in the left frontal area (channel 15) as compared with changes seen in the medium-chain triglyceride group. The left hemisphere of the brain is generally regarded to be dominant with respect to performing calculations. Our results suggest that intake of krill oil enhances the function of the cerebral hemisphere relating to calculation. A considerable decrease in oxyhemoglobin concentration changes at week 12 was observed for both tasks in the medium-chain triglyceride group. Although the reason for these results is unclear, there is a possibility that the practice effect was emphasized only in the medium-chain triglyceride condition.

Physiological measurement by near-infrared spectroscopy and EEG analyses demonstrated that krill oil and sardine oil have the effect of activating the function of the cerebral cortex. No difference was found between the three groups in terms of food-derived fatty acid intake volume. Therefore, the effects obtained in the krill oil and sardine oil groups are considered to result from ingestion of the supplements.

Both krill oil and sardine oil were shown to promote activity of the dorsolateral prefrontal cortex in terms of the working memory task. Given that these supplements contain EPA and DHA, which are not contained in medium-chain triglycerides, these fatty acids were assumed to have influenced the activity of the dorsolateral prefrontal cortex. However, sardine oil, which contains the largest amounts of these fatty acids, was not shown to possess any activation effects regarding the calculation task, and only krill oil, containing lesser amounts of these fatty acids, was shown to have an effect. The fatty acids contained in fish oil are usually stored as triglycerides. Krill oil, in contrast, has a far higher percentage of phospholipids, in particular phosphatidylcholine, than other foods. ${ }^{31-34}$ Most of the n-3 PUFAs contained in krill oil are thought to be incorporated in phosphatidylcholine. In this study, krill oil had a lower EPA and DHA content than sardine oil. Plasma EPA concentrations in the sardine oil group were significantly higher than in the medium-chain triglyceride group at week 12. On the other hand, there was no significant difference in plasma EPA concentration between the krill oil and medium-chain triglyceride groups. Nevertheless, krill oil demonstrated effects that were equivalent to or better than those of sardine oil. This led us to assume that the difference in their chemical form of incorporation was the reason for the different effects between the two oils rather than any difference in their fatty acid content.

Vaisman et al reported that ingestion of phospholipid-conjugated EPA and DHA increased the scores for a continuous performance task compared with scores obtained when the fatty acids in the ingested oil were stored as triglycerides. This finding indicates that n-3 PUFAs incorporated in phosphatidylcholine act on brain function more efficiently than those incorporated in triglycerides. ${ }^{35}$ Brossard et $\mathrm{a}^{36}$ and LemaitreDelaunay et $a{ }^{37}$ reported that lysophosphatidylcholine, which is derived from phosphatidylcholine by phospholipase $\mathrm{A}_{2}$, is the preferred physiological carrier of DHA. Thies et al found that PUFAs bound to lysophospholipids at the sn-2 position are preferentially incorporated into the brain..$^{38}$ PUFAs are most often bound to the sn-2 position in phospholipids. ${ }^{39}$ The n-3 PUFAs bound to phosphatidylcholine in krill oil may thus be taken up by the brain tissues more readily than the n-3 PUFAs of sardine oil, most likely bringing about the observed difference in their effects. However, the relationship between brain function and fatty acid composition in brain tissue has not been clarified, so further studies need to be carried out.

The major limitations of the present study are its small sample size and inclusion of male subjects only. However, its results suggest that $n-3$ PUFAs activate cognitive function of the brain in the elderly. The differences in the effects obtained by krill oil and sardine oil seem to be related to differences with regard to incorporation of fatty acids into lipids. It is concluded that fatty acids present as phosphatidylcholine have the potential to bring about more beneficial effects on cognitive function. Further studies using large sample sizes 
and including female subjects are needed to confirm the results of this study.

\section{Acknowledgment}

We thank the volunteers who participated in this study.

\section{Disclosure}

This study was funded by Nippon Suisan Kaisha Ltd. KY, KH, LH, and TT are employees of Nippon Suisan Kaisha Ltd. All authors declare that there are no other conflicts of interest in this work.

\section{References}

1. Dullemeijer C, Durga J, Brouwer IA, et al. n 3 fatty acid proportions in plasma and cognitive performance in older adults. Am J Clin Nutr. 2007;86:1479-1485.

2. Kannass KN, Colombo J, Carlson SE. Maternal DHA levels and toddler free-play attention. Dev Neuropsychol. 2009;34:159-174.

3. Muldoon MF, Ryan CM, Sheu L, Yao JK, Conklin SM, Manuck SB. Serum phospholipid docosahexaenonic acid is associated with cognitive functioning during middle adulthood. $J$ Nutr. 2010;140: 848-853.

4. Bauer I, Crewther DP, Pipingas A, Rowsell R, Cockerell R, Crewther SG. Omega-3 fatty acids modify human cortical visual processing: a double-blind, crossover study. PLoS One. 2011;6:e28214.

5. Kalmijn S, van Boxtel MP, Ocké M, Verschuren WM, Kromhout D, Launer LJ. Dietary intake of fatty acids and fish in relation to cognitive performance at middle age. Neurology. 2004;62:275-280.

6. van Gelder BM, Tijhuis M, Kalmijn S, Kromhout D. Fish consumption, n-3 fatty acids, and subsequent 5-y cognitive decline in elderly men: the Zutphen Elderly Study. Am J Clin Nutr. 2007;85:1142-1147.

7. González S, Huerta JM, Fernández S, Patterson AM, Lasheras C. The relationship between dietary lipids and cognitive performance in an elderly population. Int J Food Sci Nutr. 2010;61:217-225.

8. Jicha GA, Markesbery WR. Omega-3 fatty acids: potential role in the management of early Alzheimer's disease. Clin Interv Aging. 2010;5: 45-61.

9. Fontani G, Corradeschi F, Felici A, Alfatti F, Migliorini S, Lodi L. Cognitive and physiological effects of omega-3 polyunsaturated fatty acid supplementation in healthy subjects. Eur J Clin Invest. 2005;35: 691-699.

10. Sumich A, Matsudaira T, Gow RV, et al. Resting state electroencephalographic correlates with red cell long-chain fatty acids, memory performance and age in adolescent boys with attention deficit hyperactivity disorder. Neuropharmacology. 2009;57:708-714.

11. McNamara RK, Able J, Jandacek R, et al. Docosahexaenoic acid supplementation increases prefrontal cortex activation during sustained attention in healthy boys: a placebo-controlled, dose-ranging, functional magnetic resonance imaging study. Am J Clin Nutr. 2010;91:1060-1067.

12. Boucher O, Burden MJ, Muckle G, et al. Neurophysiologic and neurobehavioral evidence of beneficial effects of prenatal omega-3 fatty acid intake on memory function at school age. Am J Clin Nutr. 2011;93: 1025-1037.

13. Jackson PA, Reay JL, Scholey AB, Kennedy DO. DHA-rich oil modulates the cerebral haemodynamic response to cognitive tasks in healthy young adults: a near IR spectroscopy pilot study. Br J Nutr. 2012;107: 1093-1098.

14. Vakhapova V, Cohen T, Richter Y, Herzog Y, Korczyn AD. Phosphatidylserine containing omega-3 fatty acids may improve memory abilities in non-demented elderly with memory complaints: a double-blind placebo-controlled trial. Dement Geriatr Cogn Disord. 2010;29:467-474.

15. Richter Y, Herzog Y, Cohen T, Steinhart Y. The effect of phosphatidylserine-containing omega-3 fatty acids on memory abilities in subjects with subjective memory complaints: a pilot study. Clin Interv Aging. 2010;5:313-316.
16. Huppert TJ, Diamond SG, Franceschini MA, Boas DA. HomER: a review of time-series analysis methods for near-infrared spectroscopy of the brain. Appl Opt. 2009;48:D280-D288.

17. Sutton S, Ruchkin DS. The late positive complex: advances and new problems. Ann NY Acad Sci. 1984;425:1-23.

18. Kameyama M, Fukuda M, Uehara T, Mikuni M. Sex and age dependencies of cerebral blood volume changes during cognitive activation: a multichannel near-infrared spectroscopy study. Neuroimage. 2004;22:1715-1721.

19. Daltrozzo J, Wioland N, Kotchoubey B. Sex differences in two eventrelated potentials components related to semantic priming. Arch Sex Behav. 2007;36:555-568.

20. Takahashi K, Yoshimura Y, Kaimoto T, Kunii D, Komatsu T, Yamamoto S. Validation of a food frequency questionnaire based on food groups for estimating individual nutrient intake. Jpn J Nutr. 2001;59: 221-232.

21. The Council for Science and Technology, Ministry of Education, Sports, Science and Technology, Japan. Standard Tables of Food Composition in Japan. 5th revised and enlarged edition. Tokyo, Japan: National Printing Bureau; 2005.

22. Hock C, Müller-Spahn F, Schuh-Hofer S, Hofmann M, Dirnagl U, Villringer A. Age dependency of changes in cerebral hemoglobin oxygenation during brain activation: a near-infrared spectroscopy study. J Cereb Blood Flow Metab. 1995;15:1103-1108.

23. Owen AM. The functional organization of working memory processes within human lateral frontal cortex: the contribution of functional neuroimaging. Eur J Neurosci. 1997;9:1329-1339.

24. Ungerleider LG, Courtney SM, Haxby JV. A neural system for human visual working memory. Proc Natl Acad Sci U SA. 1998;95:883-890.

25. Smith EE, Jonides J. Storage and executive processes in the frontal lobes. Science. 1999;283:1657-1661.

26. Martin AJ, Friston KJ, Colebatch JG, Frackowiak RS. Decreases in regional cerebral blood flow with normal aging. J Cereb Blood Flow Metab. 1991;11:684-689.

27. Esposito G, Kirkby BS, Van Horn JD, Ellmore TM, Berman KF. Context-dependent, neural system-specific neurophysiological concomitants of ageing: mapping PET correlates during cognitive activation. Brain. 1999;122:963-979.

28. Sutton S, Braren M, Zubin J, John ER. Evoked-potential correlates of stimulus uncertainty. Science. 1965;150:1187-1188.

29. Goodin DS, Squires KC, Henderson BH, Starr A. Age-related variations in evoked potentials to auditory stimuli in normal human subjects. Electroencephalogr Clin Neurophysiol. 1978;44:447-458.

30. Pfefferbaum A, Ford JM, Kraemer HC. Clinical utility of long latency 'cognitive' event-related potentials (P3): the cons. Electroencephalogr Clin Neurophysiol. 1990;76:6-12.

31. Bottino NR. Lipid composition of two species of Antarctic krill: Euphausia superba and E. crystallorophias. Comp Biochem Physiol. 1975;50B:479-484.

32. Weihrauch JL, Posati LP, Anderson BA, Exler J. Lipid conversion factors for calculating fatty acid contents of foods. J Am Oil Chem Soc. 1977;54:36-40.

33. Kidd PM. Omega-3 DHA and EPA for cognition, behavior, and mood: clinical findings and structural-functional synergies with cell membrane phospholipids. Altern Med Rev. 2007;12:207-227.

34. Tou JC, Jaczynski J, Chen YC. Krill for human consumption: nutritional value and potential health benefits. Nutr Rev. 2007;65:63-77.

35. Vaisman N, Kaysar N, Zaruk-Adasha Y, et al. Correlation between changes in blood fatty acid composition and visual sustained attention performance in children with inattention: effect of dietary n-3 fatty acids containing phospholipids. Am J Clin Nutr. 2008;87:1170-1180.

36. Brossard N, Croset M, Normand S, et al. Human plasma albumin transports [13C]docosahexaenoic acid in two lipid forms to blood cells. J Lipid Res. 1997;38:1571-1582.

37. Lemaitre-Delaunay D, Pachiaudi C, Laville M, Pousin J, Armstrong M, Lagarde M. Blood compartmental metabolism of docosahexaenoic acid (DHA) in humans after ingestion of a single dose of $((13) \mathrm{C}) \mathrm{DHA}$ in phosphatidylcholine. J Lipid Res. 1990;40:1867-1874. 
38. Thies F, Pillon C, Moliere P, Lagarde M, Lecerf J. Preferential incorporation of sn-2 lysoPC DHA over unesterified DHA in the young rat brain. Am J Physiol. 1994;267:R1273-R1279.
39. Yamashita A, Sugiura T, Waku K. Acyltransferases and transacylases involved in fatty acid remodeling of phospholipids and metabolism of bioactive lipids in mammalian cells. $J$ Biochem. 1997;122:1-16.

\section{Publish your work in this journal}

Clinical Interventions in Aging is an international, peer-reviewed journal focusing on evidence-based reports on the value or lack thereof of treatments intended to prevent or delay the onset of maladaptive correlates of aging in human beings. This journal is indexed on PubMed Central, MedLine, the American Chemical Society's 'Chemical Abstracts

\section{Dovepress}

Service' (CAS), Scopus and the Elsevier Bibliographic databases. The manuscript management system is completely online and includes a very quick and fair peer-review system, which is all easy to use. Visit $\mathrm{http}: / /$ www.dovepress.com/testimonials.php to read real quotes from published authors.

Submit your manuscript here: http://www.dovepress.com/clinical-interventions-in-aging-journal 